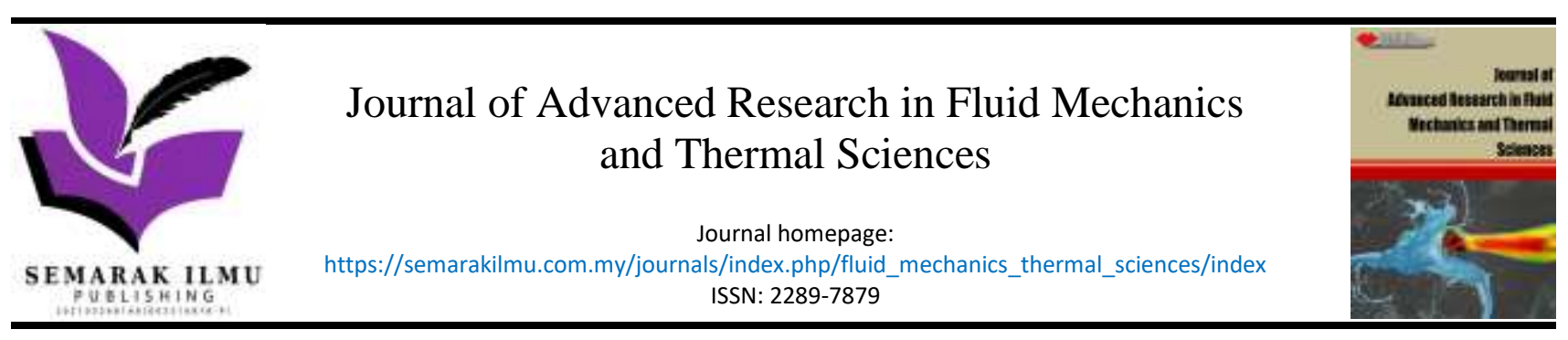

\title{
Numerical Analysis on A Non-Critical Stenosis in Renal Artery
}

\author{
Pranav Hegde ${ }^{1}$, Gowrava Shenoy B. ${ }^{1}$, A B V Barboza ${ }^{1}$, S. M. Abdul Khader ${ }^{1,}{ }^{*}$, Raghuvir Pai ${ }^{1}$, \\ Masaaki Tamagawa², Ravindra Prabhu ${ }^{3}$, D. Srikanth Rao ${ }^{1}$ \\ 1 Department of Mechanical Engineering and Manufacturing Engineering, Manipal Institute of Technology, Manipal Academy of Higher \\ Education, Manipal, 576104, Karnataka, India \\ 2 Department of Biological Functions Engineering, Graduate School of Life Science and Systems Engineering, Kyushu Institute of Technology, \\ Kitakyushu, 808-0196, Fukuoka, Japan \\ 3 Department of Nephrology, Kasturba Medical College, Manipal Academy of Higher Education, Manipal, 576104, Karnataka, India
}

\section{ARTICLE INFO ABSTRACT}

\section{Article history:}

Received 2 January 2021

Received in revised form 9 September 2021

Accepted 15 September 2021

Available online 6 November 2021

\section{Keywords:}

CFD; Renal Artery Stenosis; Angulation; Non-critical stenosis

\begin{abstract}
The increase in cardiovascular diseases worldwide has resulted in higher death rate of people globally; the primary reason being atherosclerosis. A better understanding of this condition can be achieved through the application of numerical methods to understand the haemodynamics. The present study aims to investigate the effects of renal artery angulation on the flow characteristics in a non-critically stenosed artery compared to that of a normal artery in order to understand better, the reasons for causes and progression of renal artery stenosis. Abdominal aorta-renal artery models ranging from $30^{\circ}$ to $90^{\circ}$ angulations were generated from computerized tomography-angiogram slices, post which they were subjected to cleanup and defeaturing. Haemodynamic parameters such as velocity, pressure and time-averaged wall shear stress were evaluated at early systole, peak systole and peak diastole for the different artery models. Extensive amounts of flow recirculation were observed in normal renal arteries with higher bifurcating angles, whereas it was not the case in stenosed arteries where flow acceleration was seen for the duration of the cardiac cycle. Evaluation of static pressure encountered a similar trend where an increase in angulation saw a decrease in pressure for normal arteries which contradicted with stenosed artery results. Analysis of shear stress saw very similar trends in normal and stenosed arteries, with lower angulation profiles experiencing higher values of shear stress at the Ostia. In the cases of arteries of higher angulation with a non-critical stenosis, the possibility of worsening of stenosis into an opprobrious stage remains a concern.
\end{abstract}

\section{Introduction}

According to the World Health Organization [1], the biggest cause for the number of deaths in the world are the cardiovascular diseases (CVDs). Disorders caused in the heart and blood vessels are a group of CVDs- which include coronary heart disease, cerebrovascular disease, renal artery stenosis and other diseases and is prominent amongst the elderly, usually leading to death prematurely around the age of 70 years [2]. Hypertension which is an alias for high blood pressure came to be

\footnotetext{
* Corresponding author.

E-mail address: smak.quadri@manipal.edu
}

https://doi.org/10.37934/arfmts.88.3.3148 
recognized as an essential factor for cardiovascular diseases, specifically renal artery stenosis [3]. For decades now, multiple studies have been conducted on the subject of hypertension and its propinquity with cardiovascular diseases [4]. A comparison between systolic and diastolic blood pressure as a recognition of the risk of heart disease was reviewed [5]. Atherosclerosis is the dominant cause of CVD, which include heart failure, stroke and myocardial infarction, which in the worst-case is a heart attack leading to death [6]. Atherosclerosis can be found mainly in the arteries with relatively medium to large size, such as the abdominal aorta-renal artery junction where the arteries branch out [7]. Atherosclerosis is the primary cause of Renal Artery Stenosis (RAS) [8]. It is the process of narrowing of one or both the renal arteries, which has a detrimental impact on the nephrological system as these are the arteries which carry blood to the kidneys from the aorta [9]. RAS is found to be one of the causes of hypertension and renal failure in patients $[10,11]$. It sometimes can lead to renal failure post, which the patient will have to undergo dialysis $[12,13]$. The cause of atherosclerotic renal artery stenosis (ARAS) has been generally linked by physicians with age, gender, medical conditions such as diabetes mellitus, hypertension and habits such as smoking [14]. Medical research dating back to the twentieth century has also suggested that factors such as the geometry of the arteries play a role, at least in the early development of the atherosclerotic lesions $[15,16]$.

Blood flows through different vascular systems such as coronary, abdominal and femoral systems which has a variety of curvature, bifurcations and branches in them [17]. Changes in the geometry lead to flow variation and oscillations of haemodynamic parameters such as wall shear stress, risking the chances of atherosclerosis [18]. There are various ways of diagnosis of ARAS, such as Duplex Ultrasound Diagnostics, Catheter Angiogram, Computerized Tomographic Angiography (CTA) scan, and Magnetic Resonance Angiogram (MRA) [19]. Studies done on the practicality of CTA scans found it to be an accurate tool in visualizing the images of stenosed blood vessels [20]. Clinical experiments also highlighted the use of MRA as an alternative to CTA for patients with renal insufficiency [21,22]. Treatment for RAS include the use of a stent, which is a tube designed to be inserted inside a blood vessel or a passage to keep it open in order to restore normal mass flow rate of blood [23]. For the case of atherosclerotic renal artery stenosis, primary stenting was found to be useful than the traditional clinical approach of angioplasty [24]. Other methods include the inclusion of a bypass graft which redirects the flow away from the stenosed region [25]. For the appropriate treatment of stenosis in arteries, adequate preliminary study in analyzing the haemodynamic parameters of such blood vessels are vital for appropriate treatment of stenosis [26]. While medical imaging techniques like MRA, CTA and Ultrasound as mentioned earlier can give us a proper diagnosis for these diseases, their results sometimes tend to be conflicting $[27,28]$. In such circumstances, the physician sometimes has no other option other than to diagnose based on their intuition in the matter. Therefore, the recent developments in computer technology have enabled numerical techniques to analyse the haemodynamics of cardiovascular systems and have aided doctors by acting as a complementary tool with which they can perform better diagnosis of this disease [29]. The research assessing the haemodynamic parameters due to the introduction of stents has given valuable insight into clinical treatment [30]. By creating computational models, one can acquire a better understanding of the fluid dynamics and changes in fluid parameters [31]. As a result of which, there is an increased demand for non-invasive methods like computational fluid dynamics (CFD) in the domain of biomedicine [32,33].

Recent numerical studies regarding the flow behaviour in idealized renal arteries include the analysis of fluid flow in an abdominal aorta-renal artery system. The effect of bifurcating angle of the renal artery and Reynolds number on properties such as flow velocity and wall shear stress were investigated [34]. Computational analysis, followed by an experimental examination, was done to 
find the differential pressure at the stenosed region of a renal artery [35]. The purpose of the study was to validate the accuracy of computational methods. Research on the effect of changes in the geometry of the aortoiliac region on atherosclerosis was also discussed in [36]. While studies on angulation of normal renal arteries have been carried out, the effect of geometry on a stenosed model needs to be explored. By virtue of its angulation, the risk of a non-critical stenosis can progress towards a critical stage, risking the chances of renal failure and ischemic stroke [37].

The present study focusses on the significance of renal artery angulation on idealized models. The two types of renal arteries considered are healthy and one with a single asymmetrical, non-critical stenosis. Haemodynamic variables such as flow velocity, static pressure and time-averaged wall shear stress (TAWSS) are monitored for models under the preceding cases for angles $30^{\circ}, 60^{\circ}$ and $90^{\circ}$. Several studies have highlighted the importance of such haemodynamic parameters in the early development of atherosclerosis [38-40]. This study highlights the importance of geometry as well as the consequences of renal artery stenosis (RAS). It stresses the effect of angulation on a stenosed artery compared to a normal artery; thereby, it contrives to be a complementary tool that can aid medical professionals in the diagnosis of such diseases. Numerical simulations are conducted to analyse the haemodynamic parameters for renal artery stenosis at various bifurcation angles, and the results are then compared with normal healthy arteries of similar angular profiles

\section{Methods}

\subsection{Theory}

Blood flow in this vascular model is assumed to be Newtonian, laminar and incompressible [41]. The flow is assumed to be laminar as the blood flow is simulated under resting conditions of the cardiac cycle [42]. The governing equation used is the Navier-Stokes equation given by Eq. (1) and Eq. (2) [43].

$$
\nabla \cdot v=0
$$

$\rho\left(\frac{d v}{d t}+v \cdot \nabla v\right)=-\nabla p+\mu \nabla^{2} v$

Where $\rho$ is the density, $u$ is the velocity vector, $p$ is the pressure and $\mu$ is the dynamic viscosity of the fluid.

\subsection{Modelling}

The abdominal aorta and renal artery models used in the present study were generated using the data obtained from a single central slice of a CT image. The models were subjected to geometry cleanup and defeaturing using CATIA V5 R19 [44, 45]. Renal arteries obtained where then given angulation with respect to the abdominal aortal axis at intervals of $30^{\circ}$, i.e., at $30^{\circ}, 60^{\circ}$ and $90^{\circ}$, which are referred to herein as Artery-30, Artery- 60 and Artery-90 respectively. Defeaturing of the model enables one to study the haemodynamics solely due to its geometry, where blood flow simulation is done in perfect conditions with only one parameter affecting it: bifurcation angle. Figure $1(\mathrm{a})$ and Figure 1 (b) shows the abdominal aorta models with normal and stenosed right renal artery at $30^{\circ}$ angulations. Region- 1 and 3 depict the upper and the lower parts of the abdominal aorta. Region 2 and 4 represent the right and left renal artery, with encircled region-5 on the right renal artery representing stenosis. The region of stenosis depicted in region- 5 was virtually generated with $75 \%$ stenosis, stenosis diameter 0.29 times and length of 1.125 times the renal diameter of $4 \mathrm{~mm}$. The 
stenosis is asymmetrical concerning the renal axis with an offset of $0.288 \mathrm{~mm}$. Clinical trials have proven that stenosis below the threshold of $75-80 \%$ are known to be non-critical to the functioning of the renal system $[45,47]$. It was also ascertained that $50 \%$ stenosis was the onset of decline in the arterial functions [47].

\subsection{Analysis}

The artery models were discretized using a hybrid octahedral and hexahedral mesh, as shown in Figure 1(c). Details of mesh closer to the wall to capture the effects of flow behaviour is shown in the highlighted portion. It was meshed using FLUENT 19 software, wherein the outer surface of the entire model consisted of octahedral elements and the interior, hexahedral elements; hence, the name Poly-Hexcore mesh. It is an application of mosaic technology in meshing, which results in $20-50 \%$ reduction in element count, thus speeding up the simulation process [48]. Prior to the transient simulation, grid independency tests were carried out in both the healthy and stenosed Artery-30 models. Velocity parameter was the primary flow variable monitored for different grid sizes at two sections of the model-mid upper abdominal and mid renal. The grid tests were carried out under steady state conditions, with early systolic applied at the inlet and a constant pressure of $90 \mathrm{mmHg}$ at the outlet. Figure 2(a) depicts the mesh independency test taking velocity distribution into consideration, for the healthy and single stenosed artery model. The sections monitored were the same for the two renal cases. Evaluating the grid test graphs of the normal artery model, it can be said that the parameter, i.e., velocity seems to have normalized after 350000 elements. Following the same trend were the graphs of single-stenosed artery model, where the velocity graph normalized after 400000 grid elements. Therefore, for the following analysis, a grid size of 380000 and $\mathbf{4 2 0 0 0 0}$ were considered for artery models of all three angulations under healthy and stenosed cases. The properties of blood being; density of $1050 \mathrm{~kg} / \mathrm{m}^{3}$ and dynamic viscosity of 0.00345 Pas obtained from well-known medical literatures $[49,50]$. Transient analysis was done for the modelsArtery-30, Artery-60 and Artery-90 of both cases-Healthy and Single-stenosed were done using FLUNT Solver 19.0. The pulsatile time-varying profile of velocity at the inlet, and pulsatile pressure plot displayed in Figure 2(b) was applied at the outlet in order to encompass the resistance at the periphery [22]. This plot depicts the cardiac cycle under normal resting conditions. These pulsatile profiles were assigned at the inlet and the outlet boundary conditions with the interpretation of a user-defined function. The walls of the models were assumed to be rigid [51]. The cardiac cycle which has a time period of 0.9 seconds was simulated with a total of 180-time steps, each time step of size 0.005 seconds in order to capture the flow behaviour more scrupulously [52]. Convergence criteria of $1 \times 10-5$ was employed. By analyzing the blood flow in these artery models, it is possible to ascertain the effects of angulation on healthy and stenosed abdominal aorta-renal artery models. 


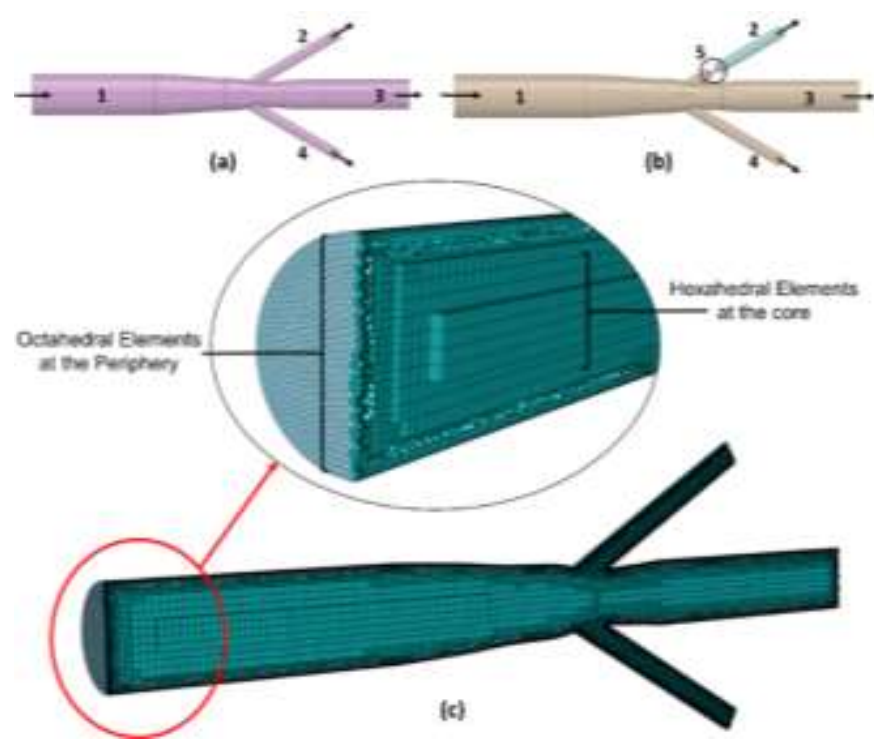

Fig. 1. Description of (a) Normal Artery-30 Model, (b) Single-Stenosed Artery-30 Model and (c) the PolyHexcore mesh

\section{a) Grid Dependency Test}
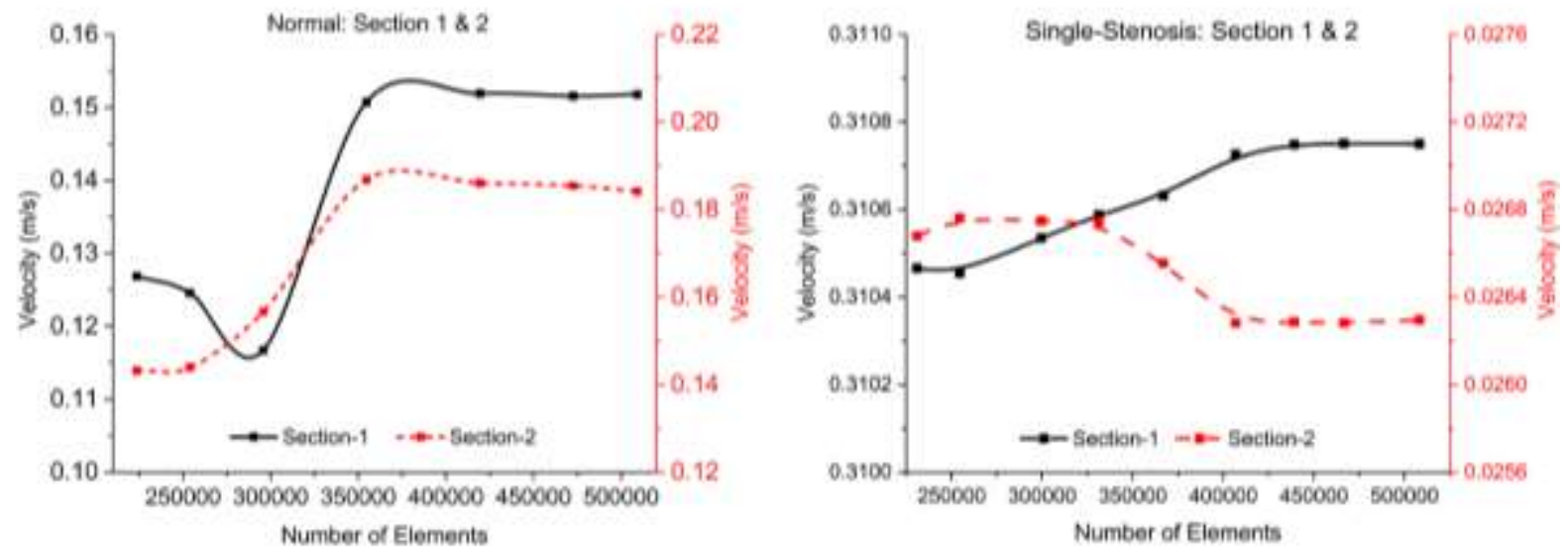

\section{b) Inlet and Outlet Boundary Conditions}
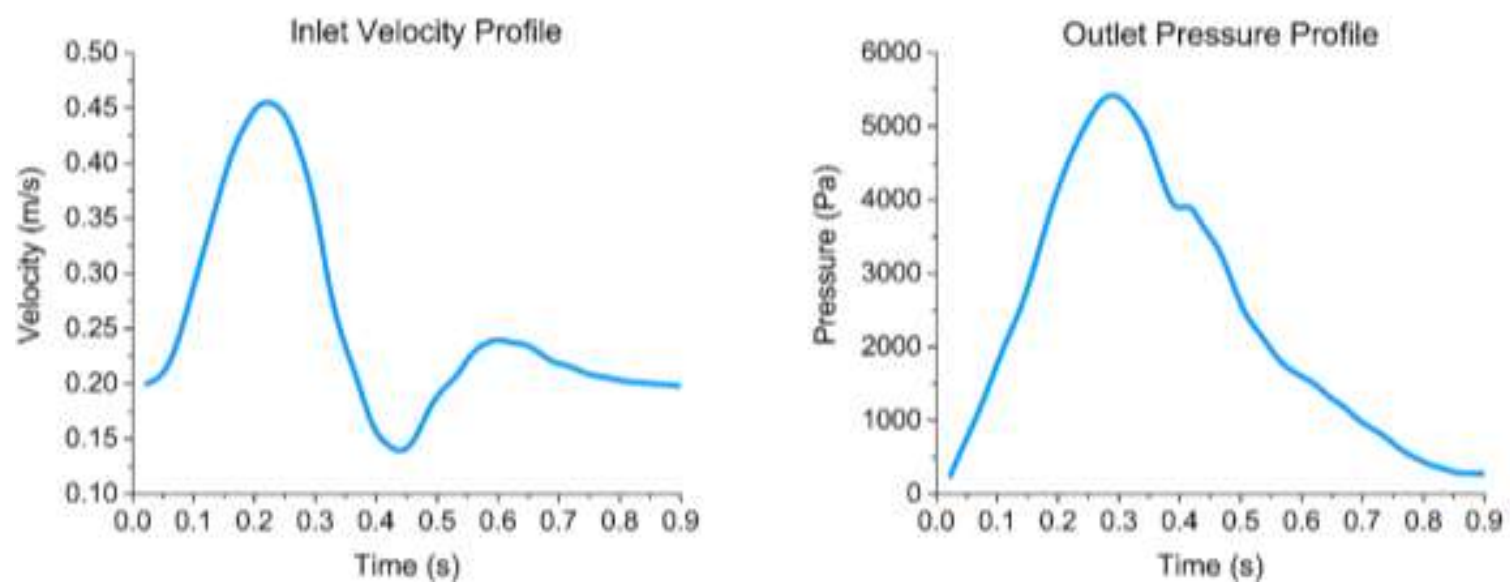

Fig. 2. (a) Grid dependency test of the Artery-30 model and (b) Inlet Velocity and Outlet Pressure profiles 


\section{Results}

Numerical analysis of healthy and stenosed artery models with three different angulations were carried out under pulsatile resting conditions. Three specific phases of the cardiac cycle; early systole $(0.06 \mathrm{~s})$, peak systole $(0.22 \mathrm{~s})$ and peak diastole $(0.44 \mathrm{~s})$ were used to investigate three haemodynamic parameters, namely flow velocity, time-averaged wall shear stress (TAWSS) and static pressure. Flow velocity determines the amount of flow recirculation in the medium. Static pressure is the pressure exerted by the static fluid on the boundary wall. These parameters are expected to vary accordingly with time due to the pulsatility of the waveform, and maximum values of parameters are generally anticipated at peak systole when the inlet velocity is maximum.

\subsection{Velocity}

The velocity contour plots of the abdominal aorta-healthy renal artery models at early systole, peak systole and peak diastole are given in Figure 3. Comparing the velocity contours at early systole, which is the beginning of the isovolumic contraction, the effect of angulation on the flow velocity was clearly observed. Apart from flow acceleration in the infrarenal region due to reduction in the geometry, it was seen that flow recirculation at the proximal end of the renal artery increased in size as the angle increased [34]. A similar trend was observed at peak systole when the inflow velocity is at the highest. Renal artery models at higher bifurcation angles experienced a lower amount of blood flow due to the increasing amount of flow obstruction. However, at peak diastole, Artery-30 experienced excessive amounts of flow recirculation in the infrarenal region. Flow in the right stenosed artery tended to be exaggerated compared to the flow in the normal arteries. Throughout the pulse cycle, it is characterized by a flow jet at the region of the stenosis or stenosed throat. At early systole, blood flow is found to accelerate at the stenosis throat, forming a jet flow as depicted in Figure 4(a). As the inlet velocity increases, so does the length of the jet, as shown in Figure 4(b), which illustrates blood flow at peak systole. Analyzing the velocity contour plots at peak diastole, given in Figure 4(c), reversed flow was observed in all the artery models, with only Artery-30 experiencing flow recirculation in the infrarenal region of the abdominal aorta. Figure 5 captures the variation in blood flow velocity along the length of the centerline of right renal artery of all the models at early systole, peak systole and peak diastole respectively. Comparing the graphs of normal and stenosed cases, it is possible to gauge the impact of angulation on the flow behaviour. The velocity with respect to time of models with normal and stenosed cases were monitored at the central point of the right renal artery. In the normal cases, arteries with lower angulations experienced higher velocities throughout the systolic phase and lower velocities in the diastolic phase, as observed in Figure 6(a). In models with stenosis, mid renal flow velocity remained lowest for Artery-30 and highest for Artery-90 throughout the pulse cycle, as observed in Figure 6(b).

\subsection{Time-averaged Wall Shear Stress}

The TAWSS parameter is a function of wall shear stress is given below, where $T$ is the period of the cardiac cycle [30].

$T A W S S=\frac{1}{T} \int_{0}^{T}|W S S| d t$ 
The plots of TAWSS calculated with a time period of 0.9 seconds; the entire duration of the cardiac cycle for normal cases and stenosed cases at various angulations are given in Figure 7(a) and Figure $7(b)$, respectively. For normal arteries, the WSS values tended to vary inversely as the bifurcation angles increased. Higher values of shear stress were observed on the distal side of the renal artery and lower values on the proximal side [53]. Comparing the TAWSS plots of stenosed arteries, shown in Figure 7(b), maximum values of shear stress was found to be at the stenosis throat, with stenosis model of Artery-90 having the highest value at that region. However, a higher value of TAWSS at the Ostia was observed for lower angulations of the renal artery. Similar to the normal arteries, distal surfaces experienced higher shear stresses compared to proximal surfaces but at higher values of WSS.

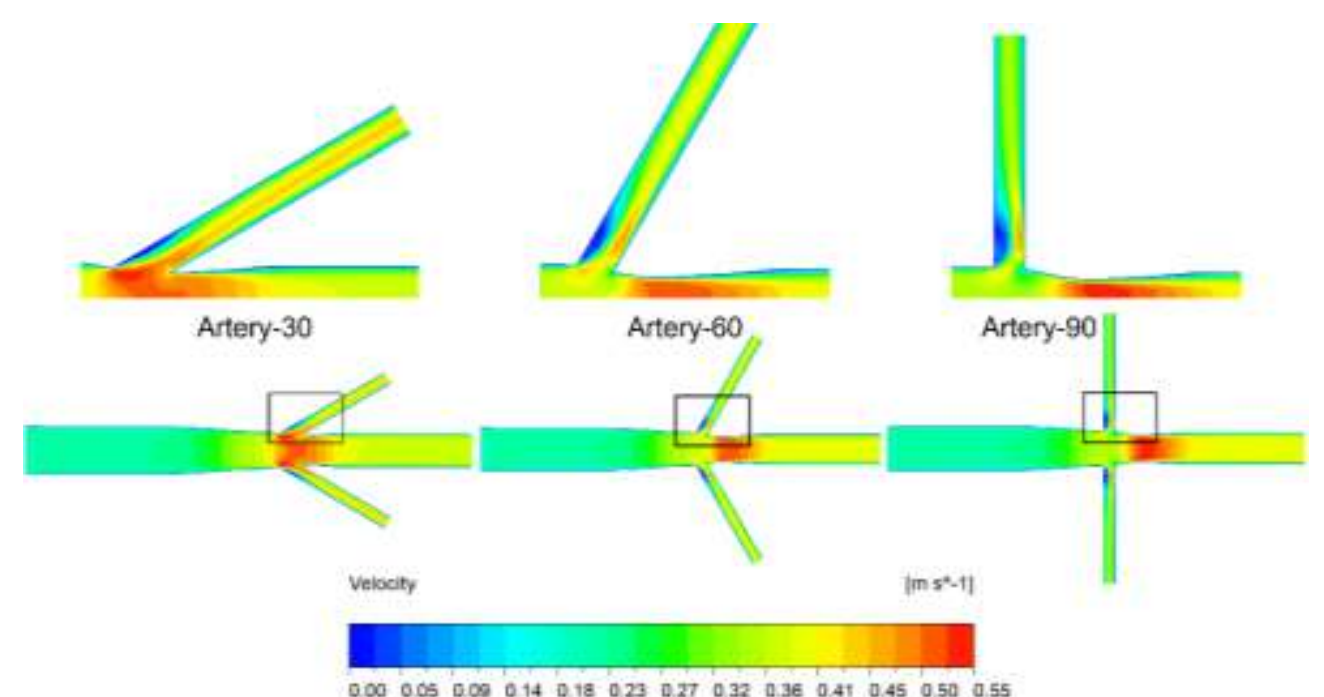

(a)

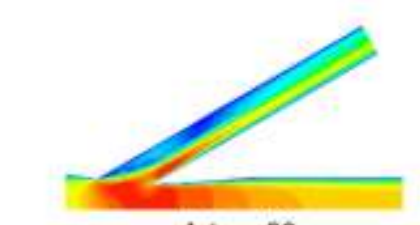

Artery-30

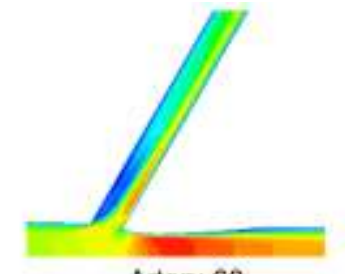

Artery-60

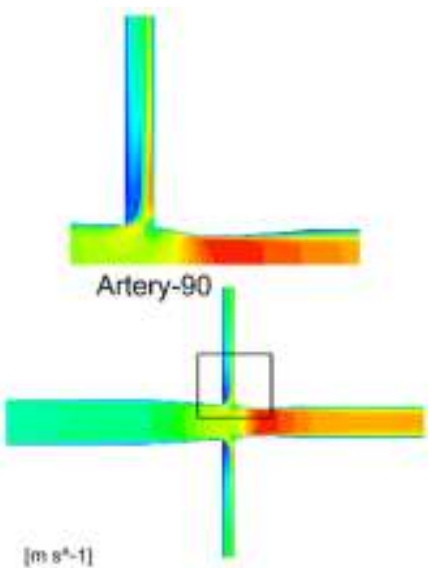

Velocily

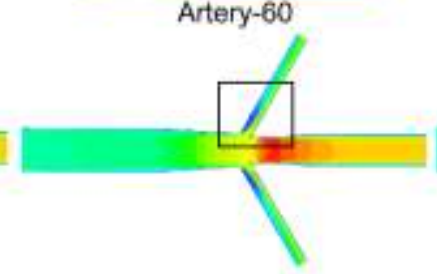

$\left[m 8^{*}-1\right]$

$\begin{array}{lllllllllllllll}0.00 & 0.09 & 0.18 & 0.27 & 0.37 & 0.46 & 0.55 & 0.84 & 0.73 & 0.82 & 0.82 & 1.04 & 1.10\end{array}$

(b) 


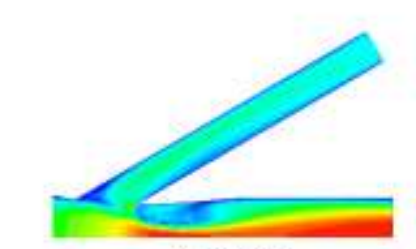

Artery-30

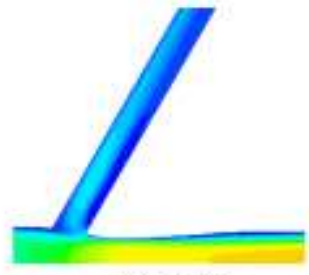

Artery-60

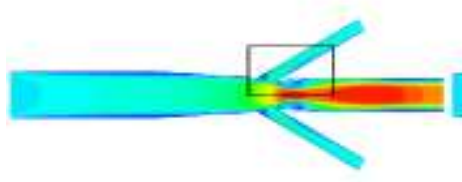

Velonity

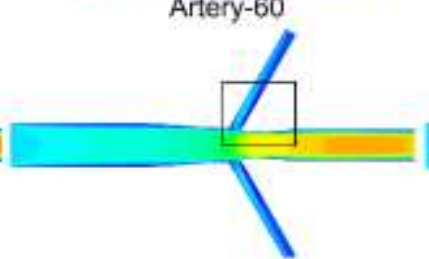

$\left[m s^{\wedge}-1 \mid\right.$

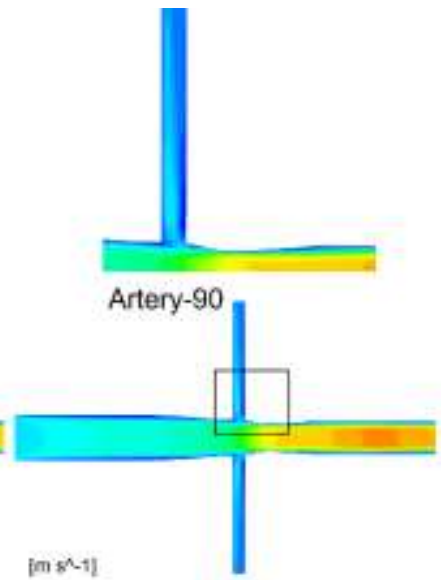

$\begin{array}{lllllllllllllllll}0.00 & 0.05 & 0.11 & 0.16 & 0.22 & 0.27 & 0.33 & 0.38 & 0.44 & 0.49 & 0.55 & 0.60 & 0.66\end{array}$

(c)

Fig. 3. Velocity contour of normal artery at (a) early systole, (b) peak systole and (c) peak diastole

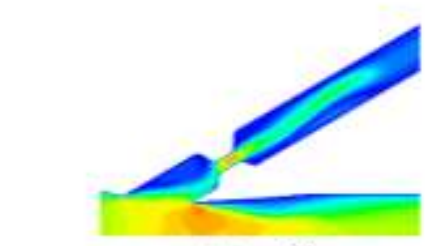

Artery-30

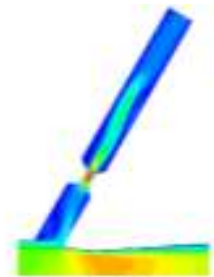

Artery-60
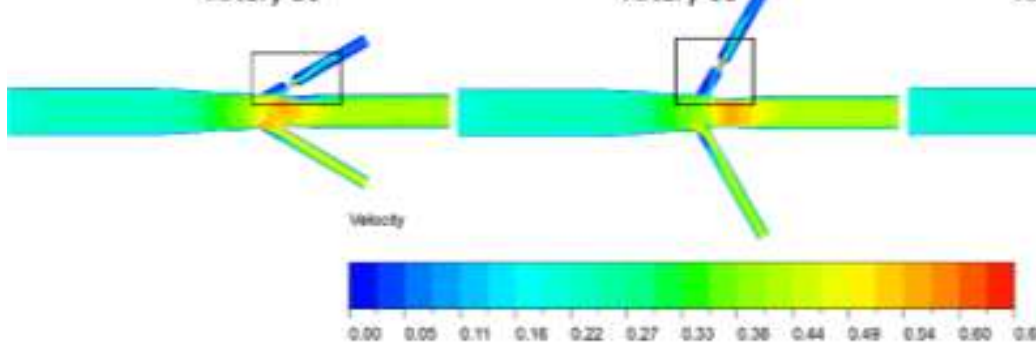

(a)

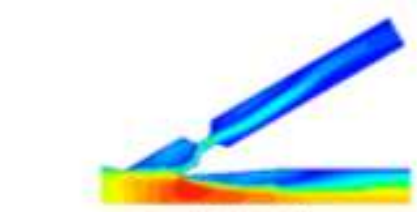

Artery-30

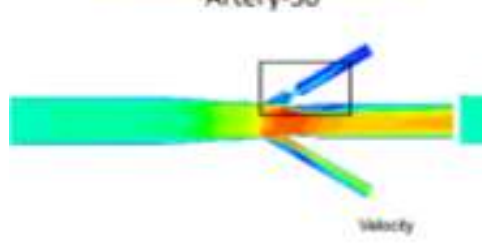

wascty
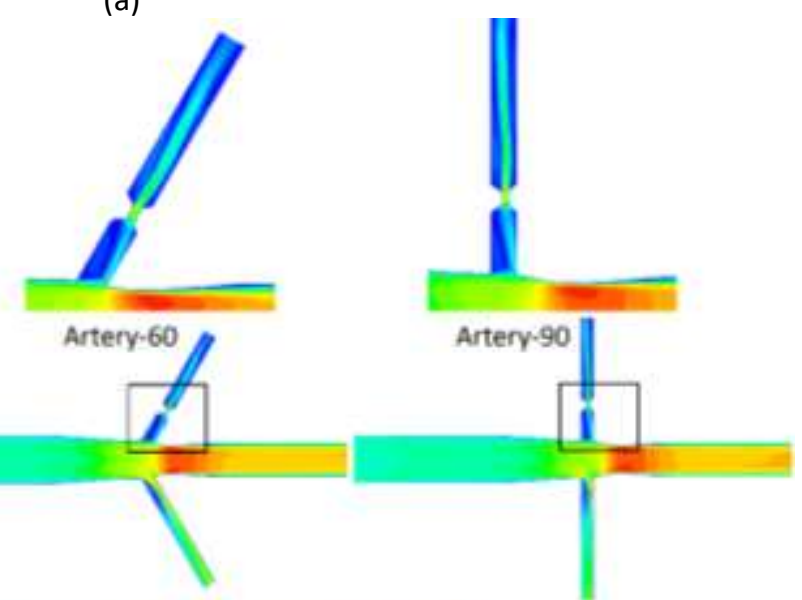

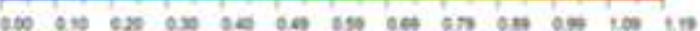

(b) 


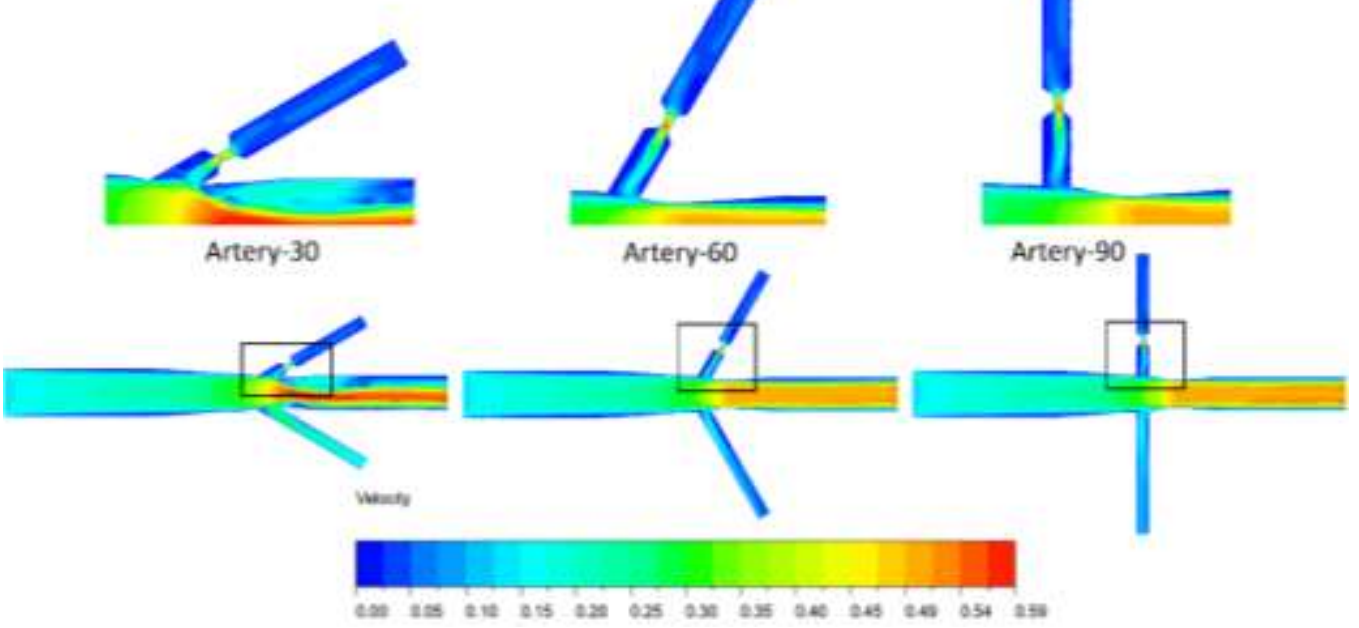

(c)

Fig. 4. Velocity contour of single-stenosed artery at a) early systole b) peak systole and c) peak diastole

\section{a) Velocity at Early Systole}
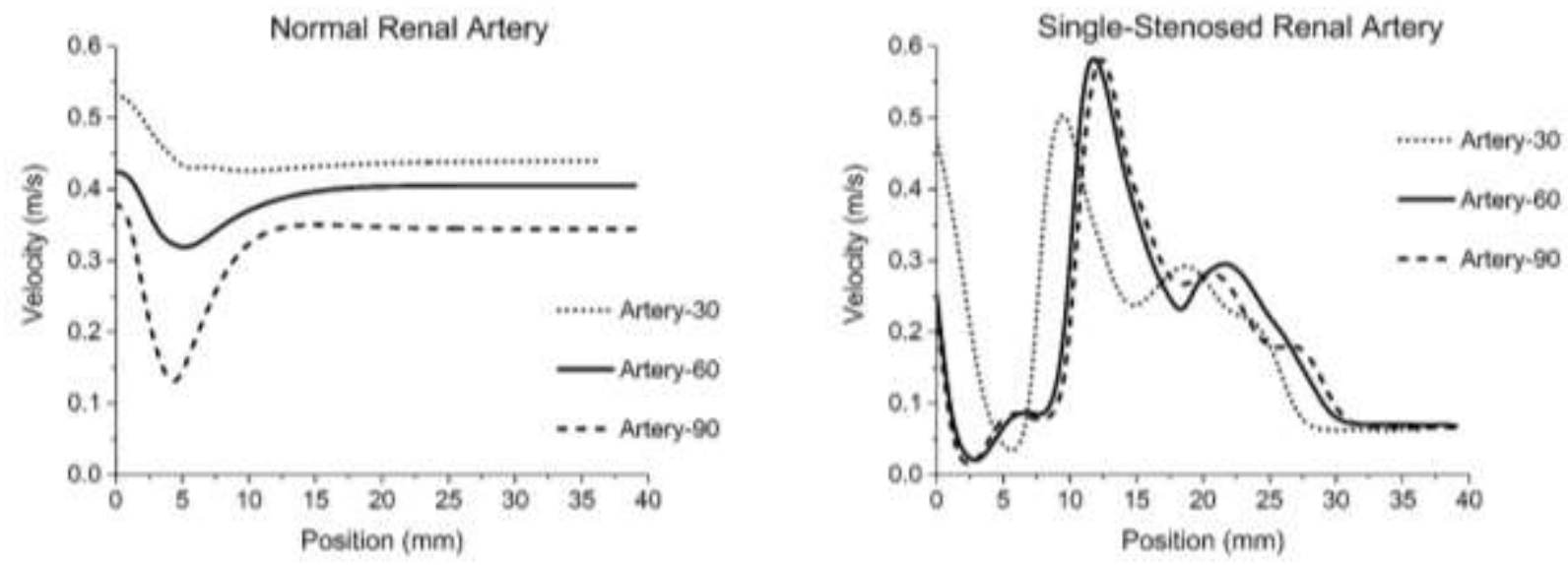

b) Velocity at Peak Systole
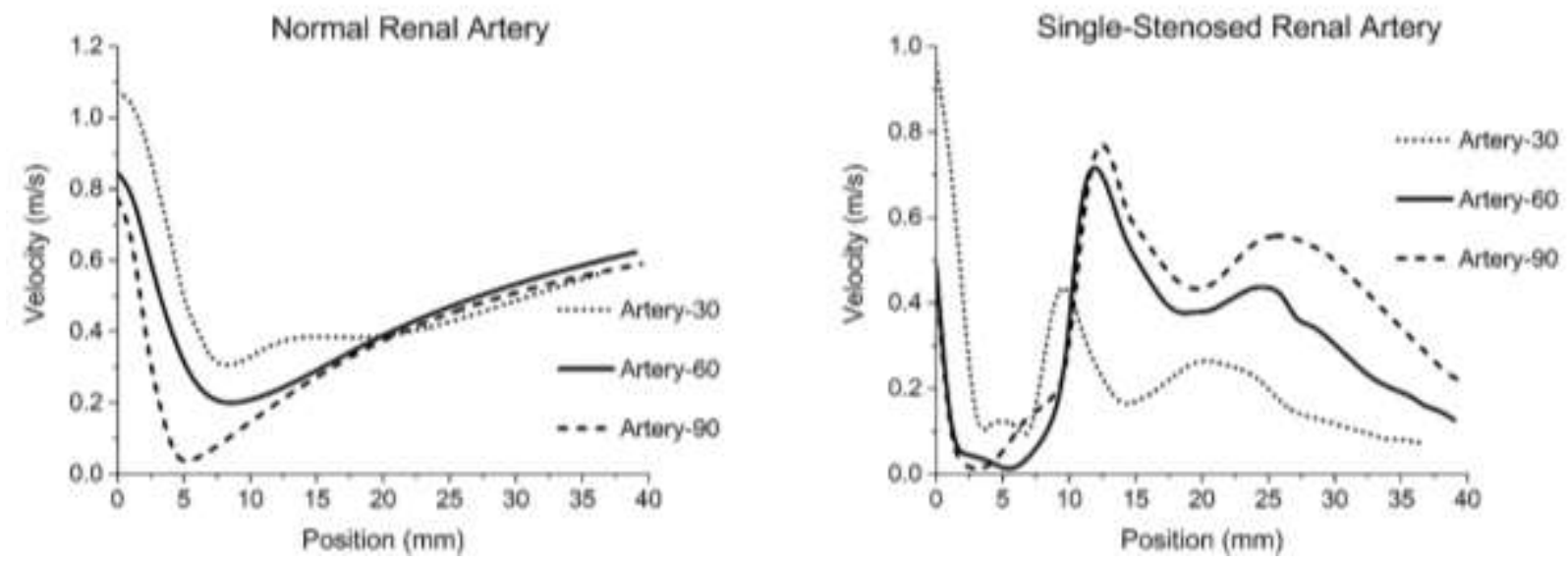


\section{c) Velocity at Peak Diastole}
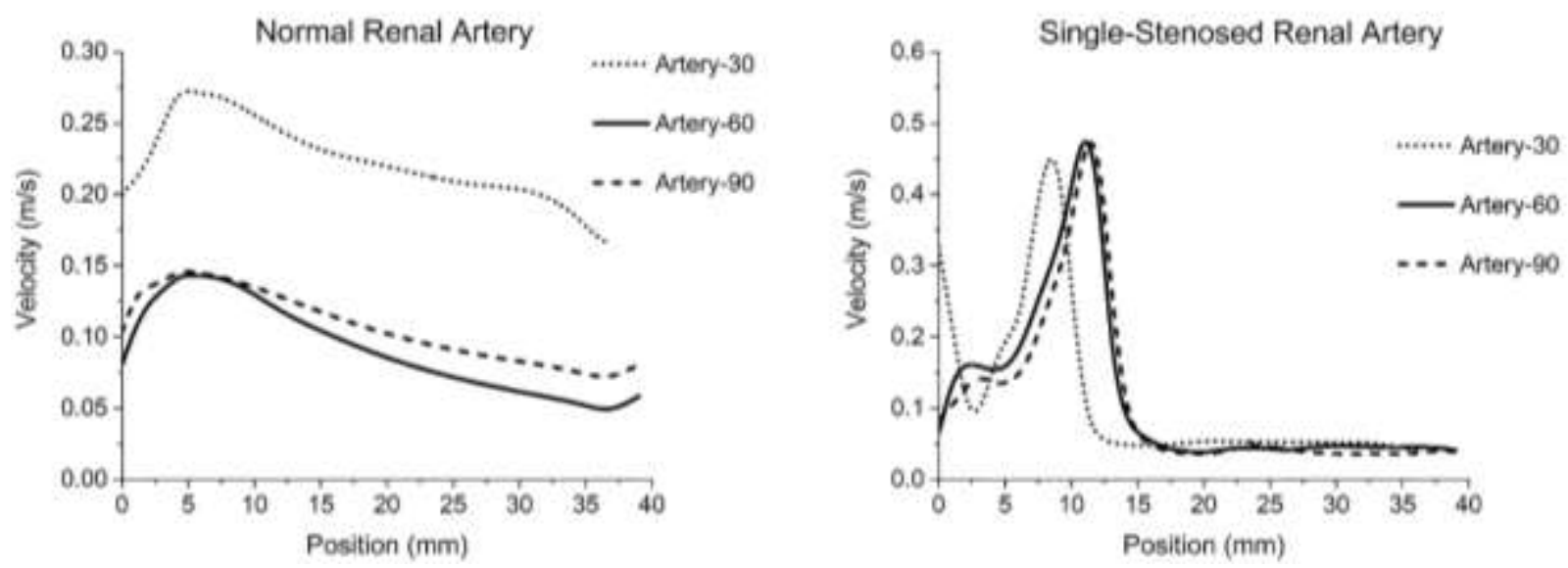

Fig. 5. Velocity along the centerline of normal and stenosed renal artery at (a) early systole, (b) peak systole and (c) peak diastole
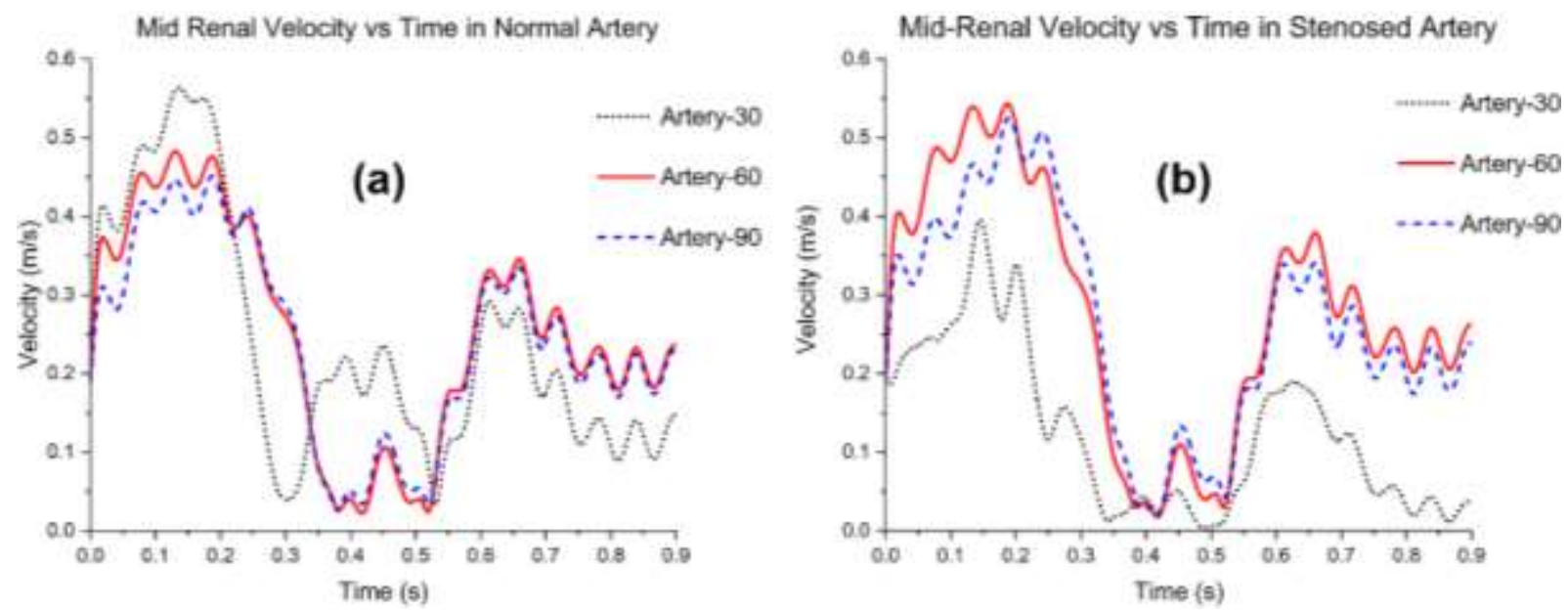

Fig. 6. Temporal variation of velocity at the mid-point of a) normal and b) stenosed renal artery

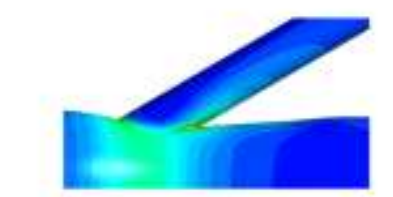

Artery-30

a) TAWSS of Normal Arteries
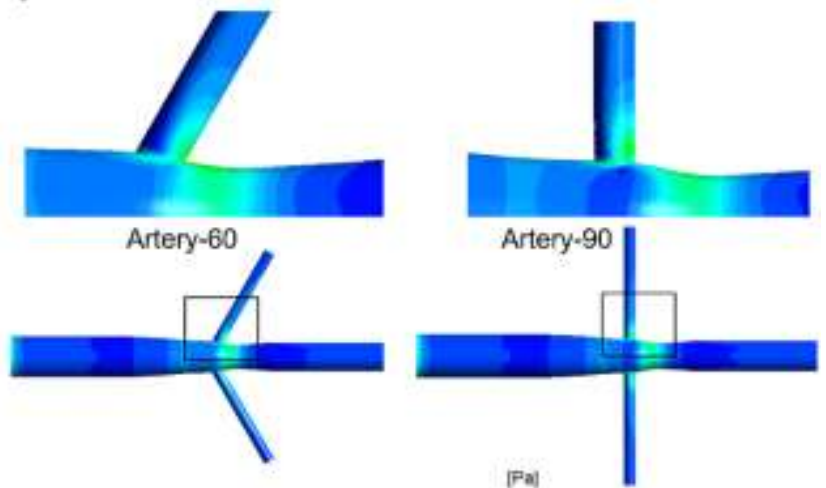

What Shear Thave

Fal

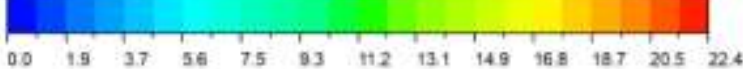


b) TAWSS of Single-Stenosed Arteries

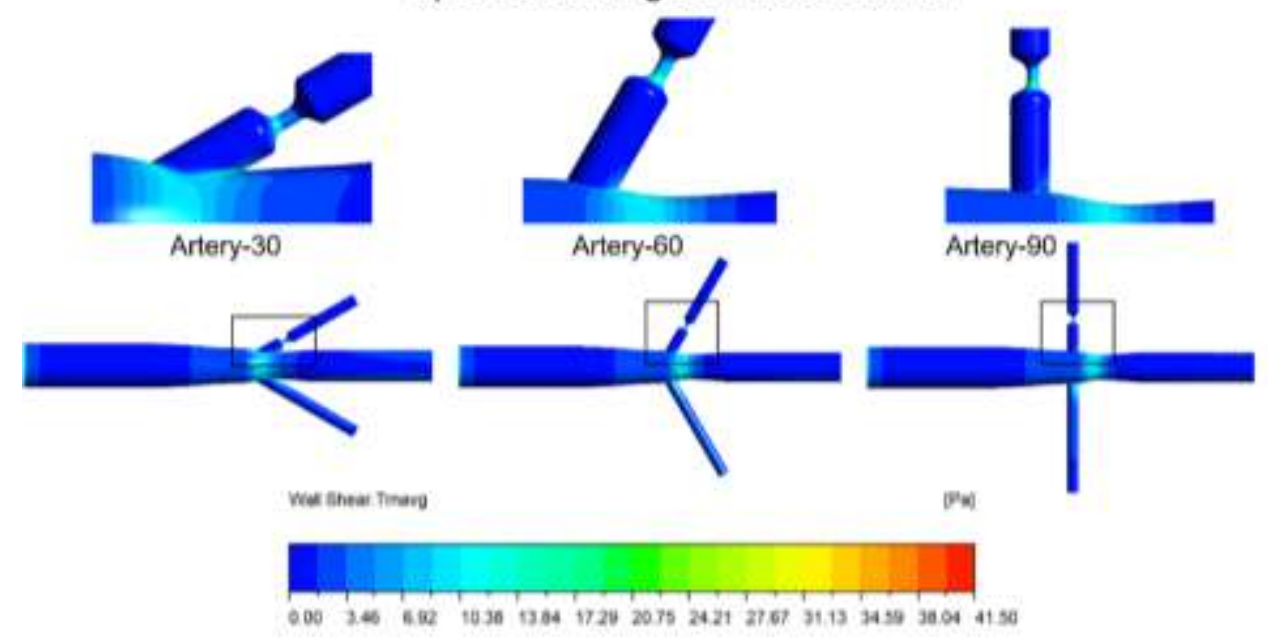

Fig. 7. TAWSS contours of a) normal and b) single-stenosed artery at the end of the pulse cycle

\subsection{Static Pressure}

Static pressure contours which describe the pressure exerted by the stationary fluid at a point in time on the rigid arterial wall. Figure 8 illustrate the static pressure contours throughout the flow medium at early systole, peak systole and peak diastole under normal cases. Positive gradient of pressure is seen in early systole as well as at peak systole. Gradual reduction in pressure along the flow is observed, albeit at peak diastole with the formation of a negative pressure gradient along the flow medium. This negative pressure gradient is what causes the backflow at peak diastole. Figure 9 describes the pressure distribution of stenosed renal arteries of various angles at early systole, peak systole and peak diastole. Pressure contour plots during the systolic phase showed a sharp pressure drop post the stenosis throat at the right renal artery for all bifurcation angles [22]. In the diastolic phase, renal and abdominal outlets had higher pressure gradients resulting in backflow, similar to normal arteries.

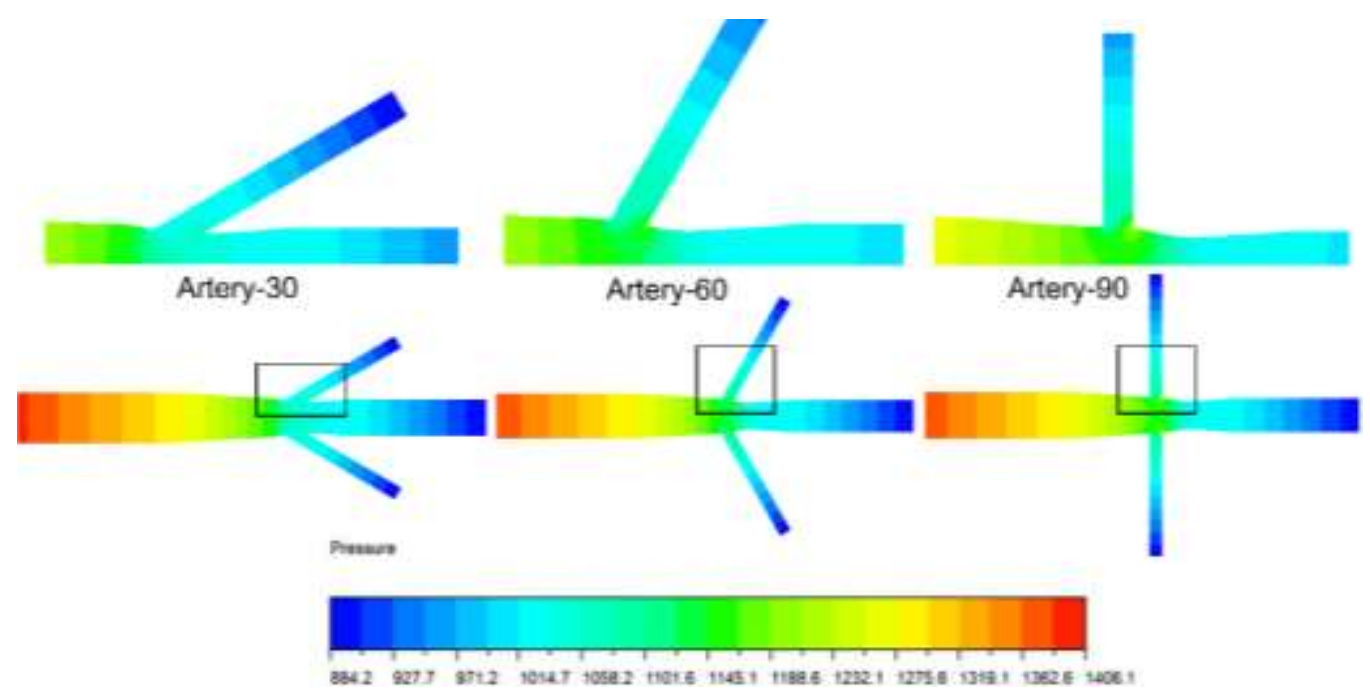

(a) 


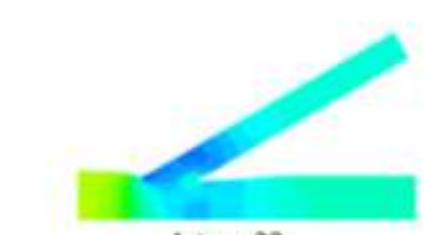

Artery-30

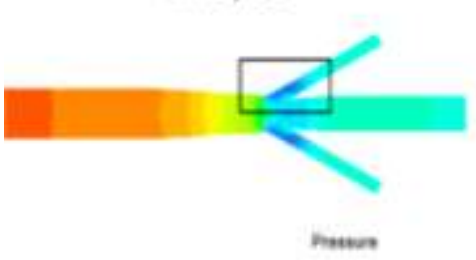

naters

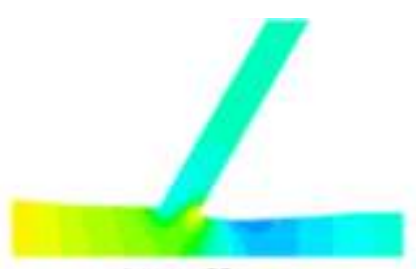

Artery-60
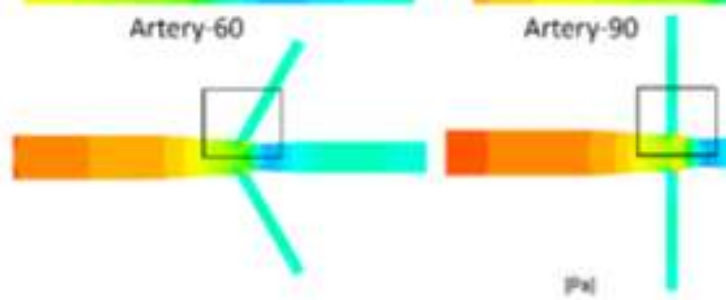

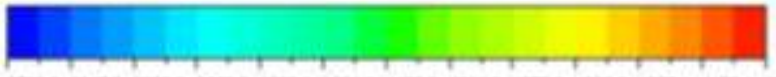

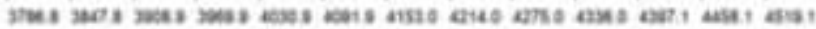

(b)

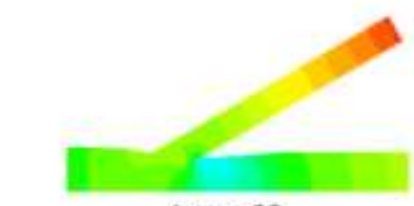

Artery-30

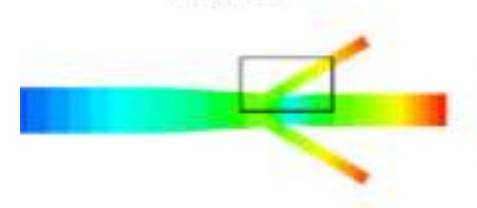

Pesare
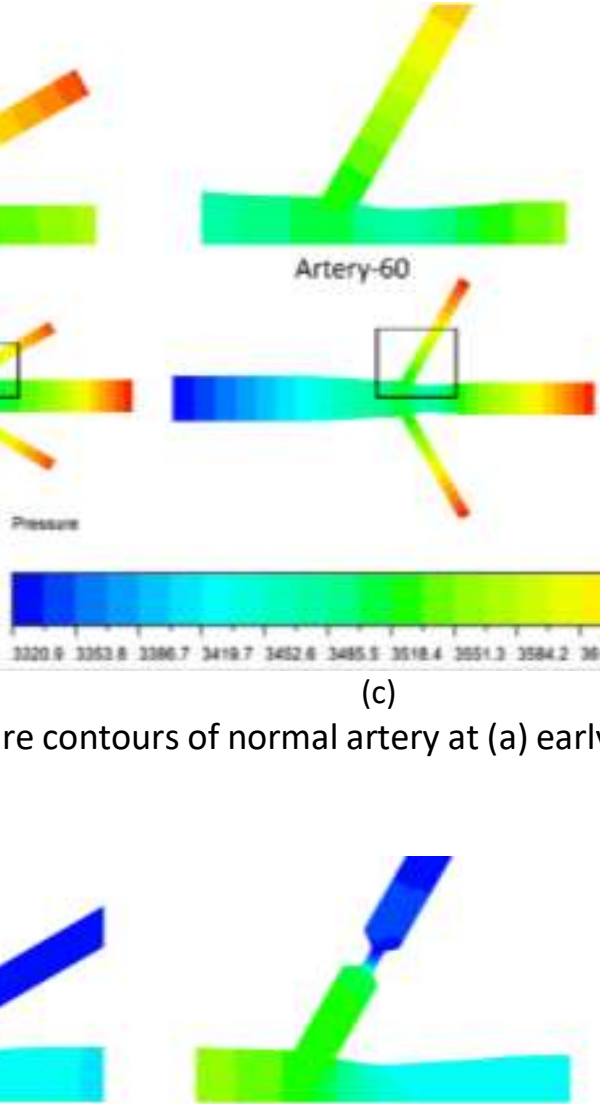

(c)
Artery-30

Artery-60

Mraner
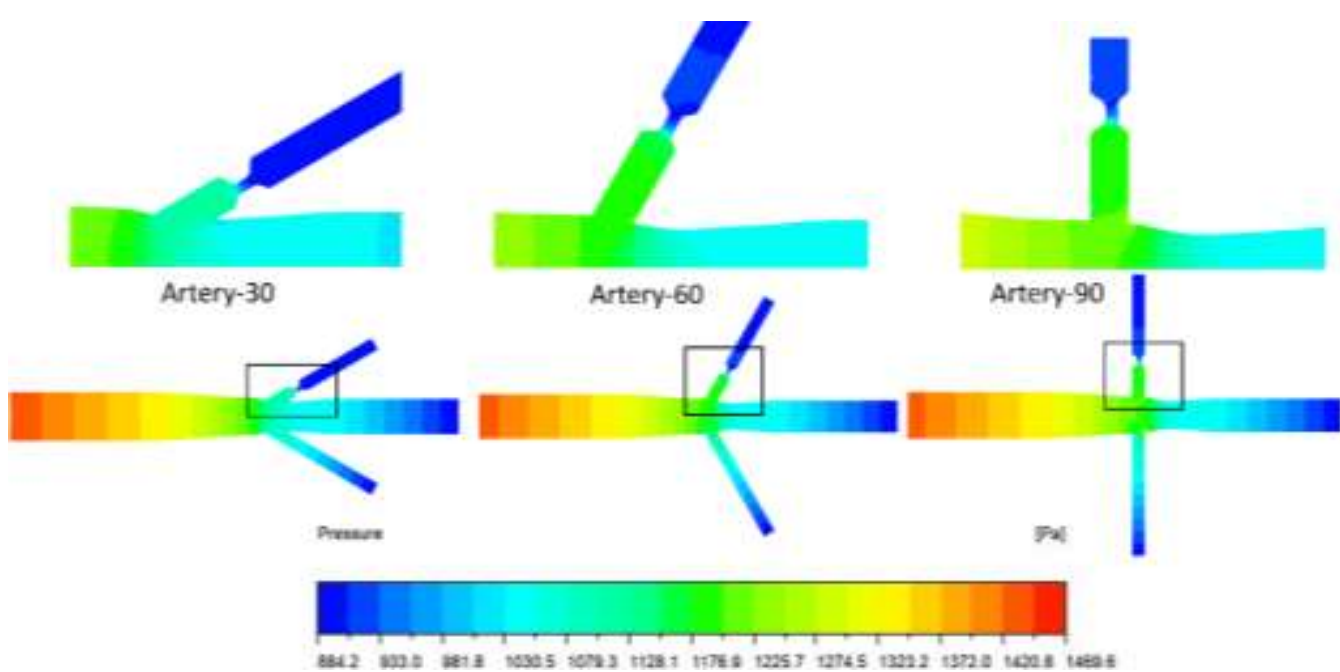

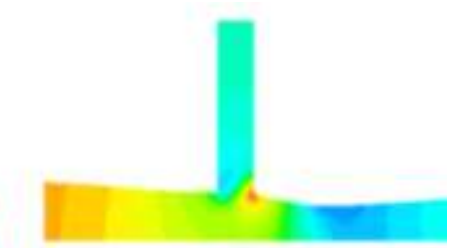

Fig. 8. Static pressure contours of normal artery at (a) early systole, (b) peak systole and (c) peak diastole

(a) 


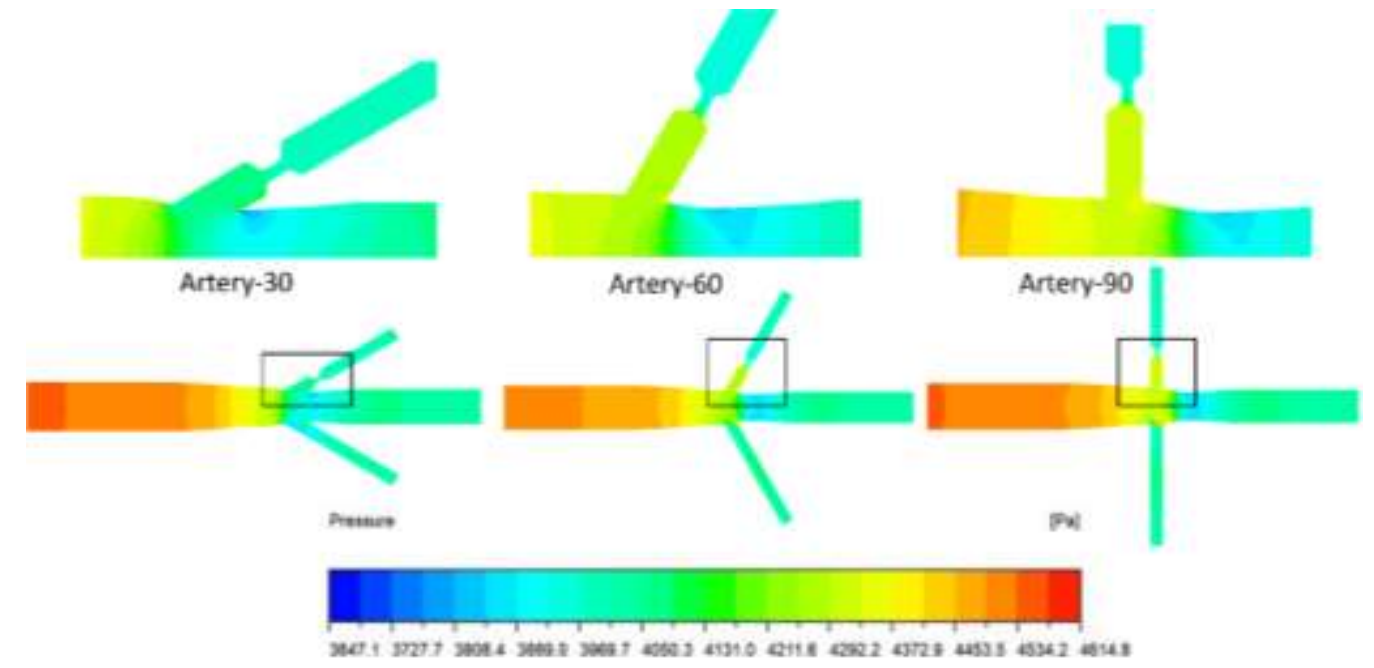

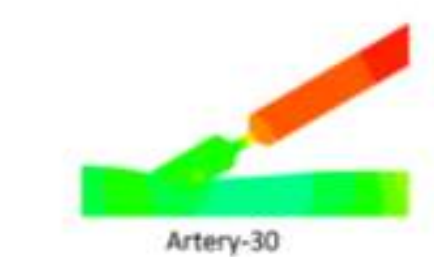

Artery-30

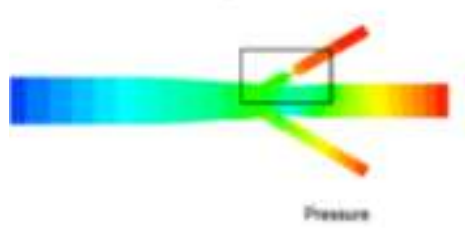

(b)

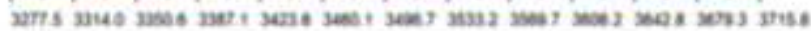

(c)

Fig. 9. Static pressure contours of single-stenosed artery at a) early systole, b) peak systole and c) peak diastole

\section{Discussions}

In this study, the relation between the geometrical characteristics of a renal artery, mainly the angulation at the Ostia and the haemodynamics of blood flow was developed. The models were taken from CT-angio patient specific renal artery data and then defeatured and idealized, in order to obtain negate the effects of other geometrical features albeit the angulation. Moreover, the effects were investigated in a renal artery with a non-critical stenosis to observe the changed in behaviour. Analysis of flow velocity saw the presence of eddies in arteries of lower angulation which was not observed in the case for arteries with higher bifurcation angles. This is due to excessive backflow from the renal artery to the abdominal aorta [54]. In all these three phases of the cardiac cycle, Artery-30 observed higher flow velocities into the renal artery. Contrary to the flow behaviour in the normal arteries, higher flow velocities were observed in arteries at a higher bifurcation angle [54]. In both instances of early and peak systole, flow at lower angulation tended to follow the curvature of the distal wall, whereas, at higher angulation of the renal artery, it impinged the surface of the distal wall which is visible in the velocity contours of stenosed Artery-60 and Artery-90 models. Further analysis of the velocity parameter was obtained by analyzing charts. This aided in examining the extent of flow recirculation due to renal artery angulation and also comparing the effects of a $75 \%$ 
stenosis. Formation of depression in the graphs in Figure 5 and Figure 6 suggest the extent of flow recirculation present in the medium. During the systolic phase of the cardiac cycle, normal arteries with lower angulation experience higher velocities and lower rates of recirculation. However, the presence of stenosis overturns this trend, as arteries with higher angulation see higher velocities, symbolized by the peak in the graphs, which represents flow acceleration [55]. Flow acceleration in relatively lower in Artery-30, but the depression at the region of the Ostia is far greater. At peak diastole, velocities in the normal case were $50 \%$ reduced when compared with that of the stenosed ones. By analyzing these factors, velocity is found to be the determining factor in the formation of eddies. Oscillation in flow patterns have also been associated with the growth of atherosclerosis [56].

Wall shear stress (WSS) is one of the most crucial parameters in the analysis of haemodynamics of blood flow, as it is the tangential force exerted by the fluid on the rigid artery wall. By averaging this value throughout a specific time period, it is possible to obtain time-averaged wall shear stress (TAWSS). Normal arteries with higher angulations experienced the shear stress distributed on a larger area of the distal surface compared to Artery-30. Artery-30 turned out to have the highest WSS values and Artery-90 the lowest. The result of a stenosis, due to increased flow velocity, resulted in higher resultant shear stress at the surfaces of the renal arteries. TAWSS presents the entire scenario of the shear stress distribution over time [57]. The regions experiencing lower wall shear stress tend to be hotspots of standing recirculation of blood throughout the cardiac cycle [58]. This leads to interaction between blood cells and the endothelial cells of the arteries resulting in the development of atherosclerosis [59]. Higher WSS values were observed at lower angulations of the renal artery for both the normal and stenosed cases. In the stenosed arteries, however, the region of the stenosed throat experienced higher amounts of WSS at higher angulations [60,61]. Increased amounts of WSS tend to cause rupture of the atherosclerotic plaque resulting in the clotting of blood in the area; resulting in complete blockage [62]. Lastly, the changes in static pressure in the medium was observed with respect to time. By analyzing this parameter, it is possible to certify the presence of backflow in a system and the extent of arterial wall thickening. Pressure parameters are inversely related with the coagulation at the walls of the artery. Normal arteries with lower angulations were found to have higher pressure regions when compared with other angles. In the single-stenosed artery models, the stenosed throat witnessed higher pressure gradients for Artery-30 compared to Artery-60 and Artery-90. The presence of a non-critical stenosis resulted in a different pressure pattern for the arteries when compared to normal arteries of similar angulations.

\section{Conclusions}

The recent developments in computational fluid dynamics (CFD) can be useful in observing the detailed haemodynamics in cardiovascular systems that can be helpful in assisting clinical evaluation and treatment. This method can have great potential in non-invasive medical diagnosis and planning. In this study, transient CFD simulations are carried out on idealistic abdominal aorta-renal artery models with a non-critical, asymmetric stenosis for different angulations of the renal artery; mainly, $30^{\circ}, 60^{\circ}$ and $90^{\circ}$. These results were then compared with the analysis results done on normal renal artery models of identical angular profiles. The haemodynamic parameters, namely flow velocity, static pressure and TAWSS were used to assess the fluid dynamics of these six models at three points of the cardiac cycle-early systole, peak systole and peak diastole. Bifurcation angles of renal arteries were found to have contrasting effects on velocity and static pressure on normal and stenosed arteries. In normal arteries, higher angular profiles were found to have greater flow recirculation regions, which was not the case in stenosed arteries. The presence of stenosis saw flow acceleration at the throat; higher velocity profile was observed in Artery-90 compared to Artery-30 throughout 
the cardiac cycle. The static pressure contours of normal arteries were distributed throughout the region with Artery-30 having a higher static pressure at the Ostia. However, the pressure at the region of stenosis saw a drastic escalation, as a result of which renal arteries with higher angulation experienced greater pressure at the Ostia. Comparing the wall shear stress parameter averaged over the time period of the entire cardiac cycle, an increase in angulation of both normal and stenosed arteries witnessed a decrease in shear stresses at the ostial region. The results show the importance of geometry in influencing the progression of atherosclerosis. It is observed that arteries with greater angular profiles were susceptible to progression of stenosis from a non-critical to a critical stage. The results obtained need to be further validated by experimental and clinical trials.

\section{References}

[1] World Health Organization. Hearts: technical package for cardiovascular disease management in primary health care. WHO Document Production Services, Geneva, Switzerland. 2016.

[2] Reddy, K. Srinath. "Cardiovascular diseases in the developing countries: dimensions, determinants, dynamics and directions for public health action." Public Health Nutrition 5, no. 1a (2002): 231-237. https://doi.org/10.1079/PHN2001298

[3] Zhu, Xiang-Yang, Victor Urbieta-Caceres, James D. Krier, Stephen C. Textor, Amir Lerman, and Lilach O. Lerman. "Mesenchymal stem cells and endothelial progenitor cells decrease renal injury in experimental swine renal artery stenosis through different mechanisms." Stem Cells 31, no. 1 (2013): 117-125. https://doi.org/10.1002/stem.1263

[4] Grant Jr, R. Peery, Ray W. Gifford Jr, William R. Pudvan, Thomas F. Meaney, Ralph A. Straffon, and Lawrence J. McCormack. "Renal trauma and hypertension." The American Journal of Cardiology 27, no. 2 (1971): 173-176. https://doi.org/10.1016/0002-9149(71)90255-4

[5] Dustan, Harriet P. "Hypertension." Journal of the American College of Cardiology 35, no. 5 S2 (2000): 10B-12B. https://doi.org/10.1016/S0735-1097(00)80064-9

[6] Swirski, Filip K., and Matthias Nahrendorf. "Leukocyte behavior in atherosclerosis, myocardial infarction, and heart failure." Science 339, no. 6116 (2013): 161-166. https://doi.org/10.1126/science.1230719

[7] Gedroyc, W. M. W., P. Neerhut, R. Negus, A. Palmer, A. Al Kutoubi, D. Taube, and B. Hulme. "Magnetic resonance angiography of renal artery stenosis." Clinical Radiology 50, no. 7 (1995): 436-439. https://doi.org/10.1016/S00099260(05)83158-7

[8] Frostegård, Johan. "Immunity, atherosclerosis and cardiovascular disease." BMC Medicine 11, no. 1 (2013): 1-13. https://doi.org/10.1186/1741-7015-11-117

[9] James, Paul A., Suzanne Oparil, Barry L. Carter, William C. Cushman, Cheryl Dennison-Himmelfarb, Joel Handler, Daniel T. Lackland et al. "2014 evidence-based guideline for the management of high blood pressure in adults: report from the panel members appointed to the Eighth Joint National Committee (JNC 8)." JAMA 311, no. 5 (2014): 507-520. https://doi.org/10.1001/jama.2013.284427

[10] Colyer Jr, William R., and Christopher J. Cooper. "Cardiovascular morbidity and mortality and renal artery stenosis." Progress in Cardiovascular Diseases 52, no. 3 (2009): 238-242. https://doi.org/10.1016/i.pcad.2009.09.004

[11] Conlon, Peter J., Krairerk Athirakul, Eugene Kovalik, Steve J. Schwab, James Crowley, Richard Stack, Charles B. McCants, Daniel B. Mark, Thomas M. Bashore, and Frank Albers. "Survival in renal vascular disease." Journal of the American Society of Nephrology 9, no. 2 (1998): 252-256. https://doi.org/10.1681/ASN.V92252

[12] Derkx, Frans, and Maarten Schalekamp. "Renal artery stenosis and hypertension." The Lancet 344, no. 8917 (1994): 237-239. https://doi.org/10.1016/S0140-6736(94)93002-3

[13] Garovic, Vesna D., and Stephen C. Textor. "Renovascular hypertension and ischemic nephropathy." Circulation 112, no. 9 (2005): 1362-1374. https://doi.org/10.1161/CIRCULATIONAHA.104.492348

[14] Arockiaa Philo Aarthy, J. "Prevalence and Predictors of Renal Artery Stenosis (RAS) in Coronary Artery Disease (CAD) Patients Undergoing Coronary Angiogram (CAG)." PhD diss., Stanley Medical College, Chennai, 2013.

[15] Friedman, Morton H., Owen J. Deters, Frank F. Mark, C. Brent Bargeron, and Grover M. Hutchins. "Arterial geometry affects hemodynamics: a potential risk factor for atherosclerosis." Atherosclerosis 46, no. 2 (1983): 225-231. https://doi.org/10.1016/0021-9150(83)90113-2

[16] Pasterkamp, Gerard, Arjan H. Schoneveld, Allard C. van der Wal, Christian C. Haudenschild, Ruud JG Clarijs, Anton E. Becker, Berend Hillen, and Cornelius Borst. "Relation of arterial geometry to luminal narrowing and histologic markers for plaque vulnerability: the remodeling paradox." Journal of the American College of Cardiology 32, no. 3 (1998): 655-662. https://doi.org/10.1016/S0735-1097(98)00304-0 
[17] Liu, Guiying, Jianhuang Wu, Dhanjoo N. Ghista, Wenhua Huang, and Kelvin KL Wong. "Hemodynamic characterization of transient blood flow in right coronary arteries with varying curvature and side-branch bifurcation angles." Computers in Biology and Medicine 64 (2015): 117-126. https://doi.org/10.1016/i.compbiomed.2015.06.009

[18] Steinman, David A. "Image-based computational fluid dynamics modeling in realistic arterial geometries." Annals of Biomedical Engineering 30, no. 4 (2002): 483-497. https://doi.org/10.1114/1.1467679

[19] Rountas, C., M. Vlychou, K. Vassiou, V. Liakopoulos, E. Kapsalaki, G. Koukoulis, I. V. Fezoulidis, and I. Stefanidis. "Imaging modalities for renal artery stenosis in suspected renovascular hypertension: prospective intraindividual comparison of color Doppler US, CT angiography, GD-enhanced MR angiography, and digital substraction angiography." Renal Failure 29, no. 3 (2007): 295-302. https://doi.org/10.1080/08860220601166305

[20] Olbricht, Christoph J., Katrin Paul, Mathias Prokop, Ajay Chavan, Cornelia M. Schaefer-Prokop, Karin Jandeleit, Karl M. Koch, and Michael Galanski. "Minimally invasive diagnosis of renal artery stenosis by spiral computed tomography angiography." Kidney International 48, no. 4 (1995): 1332-1337. https://doi.org/10.1038/ki.1995.418

[21] Sebastià, Carmen, Alejandro D. Sotomayor, Blanca Paño, Rafael Salvador, Marta Burrel, Albert Botey, and Carlos Nicolau. "Accuracy of unenhanced magnetic resonance angiography for the assessment of renal artery stenosis." European Journal of Radiology Open 3 (2016): 200-206. https://doi.org/10.1016/j.ejro.2016.07.003

[22] Zhang, Weisheng, Yi Qian, Jiang Lin, Peng Lv, Kaavya Karunanithi, and Mengsu Zeng. "Hemodynamic analysis of renal artery stenosis using computational fluid dynamics technology based on unenhanced steady-state free precession magnetic resonance angiography: preliminary results." The International Journal of Cardiovascular Imaging 30, no. 2 (2014): 367-375. https://doi.org/10.1007/s10554-013-0345-0

[23] White, C. J. "Carotid stenting vs. endarterectomy." Journal of the American College of Cardiology 64, no. 7 (2014): 722-731. https://doi.org/10.1016/i.jacc.2014.04.069

[24] Steichen, Olivier, Laurence Amar, and Pierre-François Plouin. "Primary stenting for atherosclerotic renal artery stenosis." Journal of Vascular Surgery 51, no. 6 (2010): 1574-1580. https://doi.org/10.1016/j.jvs.2010.02.011

[25] Suma, Hisayoshi, Hitoshi Fukumoto, and Atsuro Takeuchi. "Coronary artery bypass grafting by utilizing in situ right gastroepiploic artery: basic study and clinical application." The Annals of Thoracic Surgery 44, no. 4 (1987): $394-$ 397. https://doi.org/10.1016/S0003-4975(10)63799-X

[26] Strony, J., A. Beaudoin, D. Brands, and B. Adelman. "Analysis of Shear Stress and Hemodynamic Factors in a Model of Coronary Artery Stenosis and Thrombosis." The American Journal of Physiology 265, no. 5 Pt 2 (1993): H1787H1796. https://doi.org/10.1152/ajpheart.1993.265.5.H1787

[27] Schäberle, W., L. Leyerer, W. Schierling, and K. Pfister. "Ultrasound diagnostics of renal artery stenosis." Gefässchirurgie 21, no. 1 (2016): 4-13. https://doi.org/10.1007/s00772-015-0060-3

[28] Malone, Andrew, Deepa Chari, Sean Cournane, Izabela Naydenova, Andrew Fagan, and Jacinta Browne. "Investigation of the assessment of low degree $(<50 \%)$ renal artery stenosis based on velocity flow profile analysis using Doppler ultrasound: an in-vitro study." Physica Medica 65 (2019): $209-218$. https://doi.org/10.1016/j.ejmp.2019.08.016

[29] Basri, Adi Azriff, Shah Mohammed Abdul Khader, Cherian Johny, Raghuvir Pai, Muhammad Zuber, Kamarul Arifin Ahmad, and Zanuldin Ahmad. "Numerical Study of Haemodynamics Behaviour in Normal and Single Stenosed Renal Artery using Fluid-Structure Interaction." Journal of Advanced Research in Fluid Mechanics and Thermal Sciences 51, no. 1 (2018): 91-98.

[30] Martin, David M., Eoin A. Murphy, and Fergal J. Boyle. "Computational fluid dynamics analysis of balloonexpandable coronary stents: influence of stent and vessel deformation." Medical Engineering \& Physics 36, no. 8 (2014): 1047-1056. https://doi.org/10.1016/i.medengphy.2014.05.011

[31] Zhao, S. Z., X. Y. Xu, A. D. Hughes, S. A. Thom, A. V. Stanton, B. Ariff, and Q. Long. "Blood flow and vessel mechanics in a physiologically realistic model of a human carotid arterial bifurcation." Journal of Biomechanics 33, no. 8 (2000): 975-984. https://doi.org/10.1016/S0021-9290(00)00043-9

[32] Khader, Shah Mohammed Abdul, Adi Azriff, Cherian Johny, Raghuvir Pai, Mohammad Zuber, Kamarul Arifin Ahmad, and Zanuldin Ahmad. "Haemodynamics behaviour in normal and stenosed renal artery using computational fluid dynamics." Journal of Advanced Research in Fluid Mechanics and Thermal Sciences 51, no. 1 (2018): 80-90.

[33] Mandaltsi, Aikaterini, Andrii Grytsan, Aghogho Odudu, Jacek Kadziela, Paul D. Morris, Adam Witkowski, Timothy Ellam, Philip Kalra, and Alberto Marzo. "Non-invasive stenotic renal artery haemodynamics by in silico medicine." Frontiers in Physiology 9 (2018): 1106. https://doi.org/10.3389/fphys.2018.01106

[34] Ameenuddin, Mohammed, and Mohan Anand. "Effect of angulation and Reynolds number on recirculation at the abdominal aorta-renal artery junction." Artery Research $21 \quad$ (2018): 1-8. https://doi.org/10.1016/i.artres.2017.11.007

[35] Yim, Peter J., Juan R. Cebral, Ashley Weaver, Robert J. Lutz, Orlando Soto, G. Boudewijn C. Vasbinder, Vincent B. $\mathrm{Ho}$, and Peter L. Choyke. "Estimation of the differential pressure at renal artery stenoses." Magnetic Resonance in 
Medicine: An Official Journal of the International Society for Magnetic Resonance in Medicine 51, no. 5 (2004): 969977. https://doi.org/10.1002/mrm.20078

[36] Shakeri, A. B., R. S. Tubbs, Mohammadali Mohajel Shoja, H. Nosratinia, and W. J. Oakes. "Aortic bifurcation angle as an independent risk factor for aortoiliac occlusive disease." Folia Morphologica 66, no. 3 (2007): 181-184.

[37] Caps, Michael T., Claudio Perissinotto, R. Eugene Zierler, Nayak L. Polissar, Robert O. Bergelin, Michael J. Tullis, Kim Cantwell-Gab, Robert C. Davidson, and D. Eugene Strandness Jr. "Prospective study of atherosclerotic disease progression in the renal artery." Circulation 98, no. $25 \quad$ (1998): $2866-2872$. https://doi.org/10.1161/01.CIR.98.25.2866

[38] Gessner, Fred B. "Brief reviews: hemodynamic theories of atherogenesis." Circulation Research 33, no. 3 (1973): 259-266. https://doi.org/10.1161/01.RES.33.3.259

[39] Nerem, R. M., and J. F. Cornhill. "The role of fluid mechanics in atherogenesis." Journal of Biomechanical Engineering 102, no. 3 (1980): 181. https://doi.org/10.1115/1.3149571

[40] Stehbens, William E. "The role of hemodynamics in the pathogenesis of atherosclerosis." Progress in Cardiovascular Diseases 18, no. 1 (1975): 89-103. https://doi.org/10.1016/0033-0620(75)90009-2

[41] Chakravarty, S., and S. Sen. "Analysis of pulsatile blood flow in constricted bifurcated arteries with vorticity-stream function approach." Journal of Medical Engineering \& Technology 32, no. 1 (2008): 10-22. https://doi.org/10.1080/03091900600700822

[42] Valencia, Alvaro, and Francisco Solis. "Blood flow dynamics and arterial wall interaction in a saccular aneurysm model of the basilar artery." Computers \& Structures 84, no. $21 \quad$ (2006): 1326-1337. https://doi.org/10.1016/j.compstruc.2006.03.008

[43] Peskin, Charles S. "Numerical analysis of blood flow in the heart." Journal of Computational Physics 25, no. 3 (1977): 220-252. https://doi.org/10.1016/0021-9991(77)90100-0

[44] Joel, M. Elshin, and M. Anburajan. "3D Modeling of stenotic internal carotid artery treated with stent: a CFD analysis of blood." In Proceedings of the International Conference on Computer, Networks and Communication Engineering (ICCNCE 2013), vol. 30, pp. 148-151. Atlantis Press, 2013.

[45] Basri, A. A., Khader, S.M.A., Johny, C., Pai, R., Zuber, M., Ahmad, Z., Ahmad, K. A. (2020) Effect of Single and Double Stenosed on Renal Arteries of Abdominal Aorta: A Computational Fluid Dynamics. CFD Letters, 12(1), 87-97.

[46] Khader, S. M. A., Azriff, A., Johny, C., Pai, R., Zuber, M., Ahmad, K. A., Ahmad, Z. (2018) Haemodynamics Behaviour in Normal and Stenosed Renal Artery using Computational Fluid Dynamics. Journal of Advanced Research in Fluid Mechanics and Thermal Sciences, 51(1), 80-90.

[47] Simon, Geza. "What is critical renal artery stenosis? Implications for treatment." American Journal of Hypertension 13, no. 11 (2000): 1189-1193. https://doi.org/10.1016/S0895-7061(00)01179-1

[48] Andayesh, Mohammad, Azadeh Shahidian, and Majid Ghassemi. "Numerical investigation of renal artery hemodynamics based on the physiological response to renal artery stenosis." Biocybernetics and Biomedical Engineering 40, no. 4 (2020): 1458-1468. https://doi.org/10.1016/i.bbe.2020.08.006

[49] Zore, Krishna, Shoaib Shah, John Stokes, Balasubramanyam Sasanapuri, and Patrick Sharkey. "ANSYS CFD Study for High Lift Aircraft Configurations." In 2018 Applied Aerodynamics Conference, p. 2844.2018. https://doi.org/10.2514/6.2018-2844

[50] Qian, Yi, H. Takao, Mitsuo Umezu, and Yuichi Murayama. "Risk analysis of unruptured aneurysms using computational fluid dynamics technology: preliminary results." American Journal of Neuroradiology 32, no. 10 (2011): 1948-1955. https://doi.org/10.3174/ajnr.A2655

[51] Zhang, Yu, Sheau Fung Sia, Michael K. Morgan, and Yi Qian. "Flow resistance analysis of extracranial-to-intracranial (EC-IC) vein bypass." Journal of Biomechanics 45, no. $8 \quad$ (2012): $1400-1405$. https://doi.org/10.1016/i.jbiomech.2012.02.025

[52] Forrester, John H., and Donald F. Young. "Flow through a converging-diverging tube and its implications in occlusive vascular disease-I: Theoretical development." Journal of Biomechanics 3, no. 3 (1970): $297-305$. https://doi.org/10.1016/0021-9290(70)90031-X

[53] He, Yong, Nandini Duraiswamy, Andreas O. Frank, and James E. Moore Jr. "Blood flow in stented arteries: a parametric comparison of strut design patterns in three dimensions." Journal of Biomechanical Engineering 127, no. 4 (2005): 637-647. https://doi.org/10.1115/1.1934122

[54] Taylor, Charles A., Thomas JR Hughes, and Christopher K. Zarins. "Effect of exercise on hemodynamic conditions in the abdominal aorta." Journal of Vascular Surgery 29, no. 6 (1999): 1077-1089. https://doi.org/10.1016/S0741$\underline{5214(99) 70249-1}$

[55] Liang, Fuyou, Ryuhei Yamaguchi, and Hao Liu. "Fluid dynamics in normal and stenosed human renal arteries: an experimental and computational study." Journal of Biomechanical Science and Engineering 1, no. 1 (2006): 171182. https://doi.org/10.1299/jbse.1.171

[56] Mitchell, John Richard Anthony, and Colin John Schwartz. Arterial disease. Blackwell Scientific Publications, 1965. 
[57] Moore Jr, James E., Chengpei Xu, Seymour Glagov, Christopher K. Zarins, and David N. Ku. "Fluid wall shear stress measurements in a model of the human abdominal aorta: oscillatory behavior and relationship to atherosclerosis." Atherosclerosis 110, no. 2 (1994): 225-240. https://doi.org/10.1016/0021-9150(94)90207-0

[58] Fuchs, Alexander, Niclas Berg, and Lisa Prahl Wittberg. "Stenosis indicators applied to patient-specific renal arteries without and with stenosis." Fluids 4, no. 1 (2019): 26. https://doi.org/10.3390/fluids4010026

[59] Caro, C. G., J. M. Fitz-Gerald, and R. C. Schroter. "Arterial wall shear and distribution of early atheroma in man." Nature 223, no. 5211 (1969): 1159-1161. https://doi.org/10.1038/2231159a0

[60] Buchanan, J. R., C. Kleinstreuer, S. Hyun, and G. A. Truskey. "Hemodynamics simulation and identification of susceptible sites of atherosclerotic lesion formation in a model abdominal aorta." Journal of Biomechanics 36, no. 8 (2003): 1185-1196. https://doi.org/10.1016/S0021-9290(03)00088-5

[61] He, Fan, Lu Hua, and Tingting Guo. "Fluid-structure interaction analysis of hemodynamics in different degrees of stenoses considering microcirculation function." Advances in Mechanical Engineering 13, no. 1 (2021): 1687814021989012. https://doi.org/10.1177/1687814021989012

[62] Fry, Donald L. "Acute vascular endothelial changes associated with increased blood velocity gradients." Circulation Research 22, no. 2 (1968): 165-197. https://doi.org/10.1161/01.RES.22.2.165 